\title{
A 3D model to predict the influence of nanoscale pores or reduced gas pressures on the effective thermal conductivity of cellular porous building materials
}

\author{
Wouter Van De Walle ${ }^{1}$, Hans Janssen ${ }^{1}$ \\ ${ }^{1} \mathrm{KU}$ Leuven, Department of Civil Engineering, Building Physics Section, Kasteelpark Arenberg 40, 3001 \\ Heverlee (Belgium).
}

wouter.vandewalle@kuleuven.be; hans.janssen@kuleuven.be

\begin{abstract}
Cellular porous materials are frequently applied in the construction industry, both for structural and insulation purposes. The progressively stringent energy regulations mandates the development of better performing insulation materials. Recently, novel porous materials with nanopores or reduced gas pressures have been shown to possess even lower thermal conductivities because of the Knudsen effect inside their pores. Further understanding of the relation between the pore structure and the effective thermal conductivity is needed to quantify the potential improvement, and design new optimized materials. This paper presents the extension of a 3D numerical framework simulating the heat transfer at the pore scale. A novel methodology to model the reduced gas-phase conductivity in nanopores or at low gas pressures is presented, accounting for the three-dimensional pore geometry while remaining computationally efficient. Validation with experimental and numerical results from literature indicates the accuracy of the methodology over the full range of pore sizes and gas pressures. Combined with an analytical model to account for thermal radiation, the framework is applied to predict the thermal conductivity of a nanocellular PMMA foam experimentally characterized in literature. The simulation results show excellent agreement with less than $5 \%$ difference with the experimental results, validating the model's performance. Furthermore, results also indicate the potential improvements when decreasing the pore size from the micrometre to the nanometre range, mounting up to $40 \%$ reduction for such high-porosity low-matrix-conductivity materials. Future application of the model could assist the design of advanced materials, properly accounting for the effect of reduced pore sizes and gas pressures.
\end{abstract}

\section{Nomenclature}

\begin{tabular}{|lll|}
\hline Greek & thermal conductivity & $\mathrm{W} / \mathrm{mK}$ \\
$\lambda$ & mass density & $\mathrm{kg} / \mathrm{m}^{3}$ \\
$\rho$ & Stefan-Boltzmann constant & $\mathrm{W} / \mathrm{m}^{2} \mathrm{~K}^{4}$ \\
$\sigma$ & Emissivity & - \\
$\epsilon$ & & \\
& & $\mathrm{K}$ \\
Roman & Temperature & - \\
$T$ & Net transmittance through a unit cell & $\mathrm{W} / \mathrm{m}^{2}$ \\
$T_{\text {net }}^{\text {unit }}$ & Heat flux & $\mathrm{m}{ }^{3}$ \\
$q$ & Volume & $\mathrm{J} / \mathrm{kgK}$ \\
$V$ & Specific heat capacity at constant volume & $\mathrm{J} / \mathrm{m}^{3} \mathrm{~K}$ \\
$C_{V}$ & Heat capacity per volume for thermal radiation & - \\
$C_{\text {rad }}$ & Radiation factor & - \\
$C\left(\epsilon, S_{f}\right)$ & & \\
\hline
\end{tabular}




\begin{tabular}{|lll|}
\hline$S_{f}$ & Slenderness factor & - \\
$v$ & Speed & $\mathrm{m} / \mathrm{s}$ \\
$L$ & Length & $\mathrm{M}$ \\
$R$ & Specific gas constant & $\mathrm{J} / \mathrm{kgK}$ \\
$p$ & Pressure & $\mathrm{Pa}$ \\
$d$ & Thickness & $\mathrm{m}$ \\
$d_{x}$ & Mean distance in the x-direction & $\mathrm{m}$ \\
$a$ & Thermal accommodation coefficient & - \\
& & \\
Abbreviations \& Acronyms & & $\mathrm{W} / \mathrm{mK}$ \\
ETC & Effective thermal conductivity & \\
eff & effective & \\
cond & conductive & \\
rad & radiative & \\
x,y or z & in the x, y or z-direction & \\
MFP & Mean free path & \\
avg & Average & \\
bdy & boundary & \\
\hline
\end{tabular}

\section{Introduction}

Heat losses through opaque building components like walls and roofs account for a large part of the total heating demand in buildings. Hence, porous insulation materials are often applied, offering a relatively low thermal conductivity due to their porous nature (Jelle, 2011; Schiavoni et al., 2016; Smith et al., 2013). Examples are insulation foams like XPS and PUR, mineral insulations like stone and glass wool, but also materials combining structural and insulating properties like aerated concrete or cellular glass. Complying with the progressively stringent energy regulations requires increasingly thick insulation layers, complicating the design and the refurbishment of new and existing buildings. A lot of research is hence focussed on obtaining better performing insulations materials, with effective thermal conductivities below that of still air $\left(\sim 25 \mathrm{~mW} / \mathrm{mK}\right.$ at $\left.20^{\circ} \mathrm{C}\right)$.

One of the possible strategies is optimizing the pore structure parameters, since they directly influence the materials' effective thermal conductivity (ETC). Indeed, the ETC originates from several heat transfer mechanisms at the microscale, most importantly being heat conduction through the solid matrix and through the gas, and thermal radiation through the material. To reduce the ETC, typical insulation materials have very elevated porosities, at which conduction through the gas phase accounts for the largest part of the total heat transfer (Solórzano et al., 2009). Hence, research has been focussing on reducing the contribution of the gas-phase conductivity. Several possible pathways were identified by Jelle et al. (2010): replacing the gas with a less conductive gas, reducing the gas pressure or confining the gas in nanoscale pores. The latter two strategies show great application potential for building insulation purposes, with examples like vacuum insulation panels, aerogels, nanocellular foams and hollow silica nanospheres (HSNS) (Baetens et al., 2010; Cuce et al., 2014; Hu et al., 2018; Liu et al., 2015). For such materials, it has been shown that the gas-phase conduction mechanism shifts from the Fourier to the Knudsen regime, resulting indeed in a reduction of the gasphase conductivity. Further optimization of these novel materials and design of new materials requires a better understanding though of the combined impact of the pore structure parameters like the porosity, pore shape or pore size distribution for example on the resulting ETC.

Large-scale experimental production and characterization of such novel materials with different properties is very cumbersome though. Therefore, different modelling strategies have been 
developed to advance the knowledge and understanding of porous materials with nanosized pores or low gas pressures. Analytical expressions, on one hand, allow predicting the ETC as a function of several pore scale parameters like porosity and pore size. Usually, a separate conductivity is derived for each relevant heat transfer mechanism based on empirically or theoretically derived correlations. Material characteristics like porosity or pore size are averaged to use in these correlations. Subsequently, the thermal conductivities of the different heat transfer mechanisms are combined by summation or through other formulations to obtain the ETC. Examples and reviews for different types of materials can be found in literature (Ferkl et al., 2013; He and Xie, 2015; Hu et al., 2018; Kan et al., 2015; Machrafi and Lebon, 2015; Wang, 2017a). Such analytical expressions provide very useful insights regarding the expected impact of certain parameters. Indeed, Wang et al. (2017a) for example demonstrated the potential existence of an optimum porosity in nanocellular foams at which the ETC reaches a minimum value. However, since such models are based on averaged properties, they don't allow studying the effect of parameters like the pore size distribution width or a locally varying pore shape. Furthermore, their validity and applicability for the whole porosity range or diverse pore structures is unclear. On the other hand, several highly detailed modelling strategies have been developed to study the effect of the gas pressure or the dimensions of the pore size and/or matrix walls. Examples are the molecular dynamics technique, phonon Boltzmann equation techniques and phonon hydrodynamics (Bao et al., 2018; Coquil et al., 2011; Guo and Wang, 2015). Very detailed information on the effect of several pore scale parameters can be gained from such models. However, they are currently still computationally very expensive, implying that they can only be used for modelling small or simplified configurations. This impedes their application for modelling a representative volume of the specimen or performing optimization efforts which require simulations of extended sample sets.

This paper discusses the extension of an existing 3D image-based thermal conductivity model (Van De Walle et al., 2018) to simulate porous materials containing nanosized pores or with low gas pressures. The model performs simulations on the 3D pore structure of the material, inherently incorporating the effect of the pore structure parameters. An efficient methodology to include the effect of the local pore size, shape and gas pressure on the gas-phase conductivity is presented. The model framework is validated comparing the simulation performance with experimental measurements found in literature for a set of nano- and microcellular foams. The extended model framework hence aims at further understanding the effect of the pore structure on the ETC of porous materials, including the potential impact of reduced pore sizes or gas pressures.

The model presented is aimed primarily at predicting the thermal conductivity of cellular materials, commonly found in typical porous building materials. Indeed, such pore structure topologies have been shown to combine low thermal conductivities with strong structural properties (Ashby and Medalist, 1983; Gibson and Ashby, 1997). Using the model presented in this paper, the authors hope to contribute to the further understanding of the thermal properties of cellular porous materials with application in the building industry.

\section{Extension of the model framework}

Recently, the authors presented an image-based model to study the effect of the pore structure parameters on the ETC in macro-cellular materials like cellular concrete or cellular glass (Van De Walle et al., 2018). Further thermal optimization of such materials could be achieved by reducing the pore size and/or the gas pressure as was discussed earlier. Therefore, this section discusses the extension of the model framework to incorporate the effect of such adaptations on the resulting ETC. Since these 
modifications mainly target the reduction of the gas-phase conductivity, focus is placed on formulating a model strategy for this heat transfer mechanism. A calculation methodology quantifying the gasphase conductivity in every pore based on its local dimensions, shape and gas pressure is derived and validated with experimental measurements of the conductivity of some gasses found in literature. Subsequently, an analytical model proposed in literature (Wang et al., 2018; Wang, 2017a) to account for thermal radiation is revisited, before discussing the conduction through the solid matrix.

\subsection{Image-based prediction model}

A brief overview of the original 3D image-based model framework extended in this paper is presented here. The framework performs simulations on a cubic sample of the material and consists of three main parts. An illustrative overview is shown in Figure 1.

1. Geometry

2. Meshing

3. Simulation
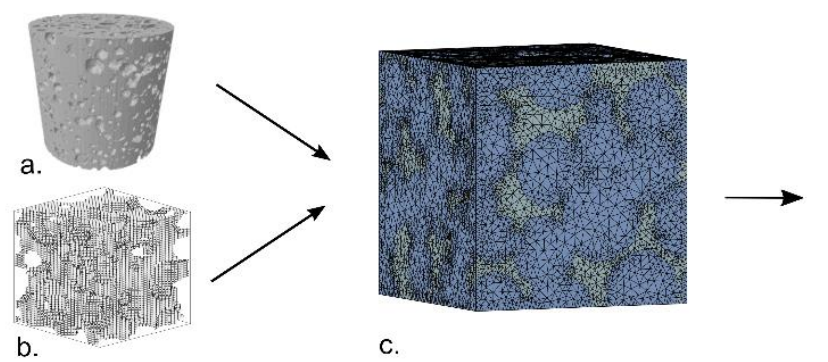

c.

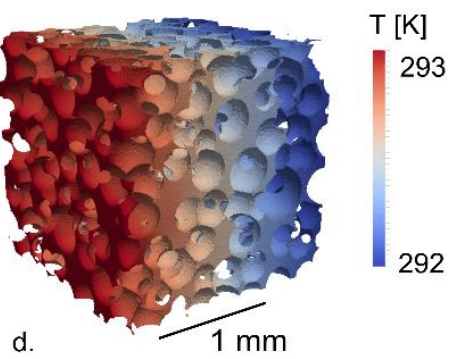

Figure 1: Overview of model framework with (a) a $\mu$ CT image of an aerated concrete, $(b)$ a virtually generated cellular material, (c) the finite element mesh and (d) the temperature profile after the simulation (Van De Walle et al., 2018).

First, a 3D representation of the pore structure needs to be obtained. This can be either through imaging techniques like $\mu \mathrm{CT}$ scanning or through virtual generation algorithms. The image format is a $3 \mathrm{D}$ cubic voxel image matrix with side length $d_{\text {sample }}[\mathrm{m}]$. Subsequently, a finite element mesh is built directly on the 3D image using an adaptation of the open source codes iso2mesh and CGAL (Alliez et al., 2015; Fang and Boas, 2009). Finally, based on this mesh, a stationary thermal simulation is performed in a simulation package like COMSOL Multiphysics or Elmer. A constant temperature difference is applied across the sample and all other boundaries are set to adiabatic conditions. The thermal conductivity of the matrix phase and the gaseous phase are set to their specified values found in literature. The effective thermal conductivity of the sample is derived from the heat flow results of the simulation through the formula in Eq. 1, with $q_{x}\left[\mathrm{~W} / \mathrm{m}^{2}\right]$ the heat flux in the general heat transfer direction, $T_{\text {hot }} / T_{\text {cold }}[K]$ the applied boundary temperatures and $d_{\text {sample }}[\mathrm{m}]$ the side length of the simulated cubic sample (Chen et al., 2015). All simulations are performed assuming dry conditions, in accordance with the usual practice of measuring a material's thermal conductivity in dry conditions.

$$
\lambda_{\text {eff }}=\frac{\int_{V_{\text {cube }}} q_{x} * d V}{\left(T_{\text {hot }}-T_{\text {cold }}\right) *\left(d_{\text {sample }}\right)^{2}}
$$

Thermal radiation is taken into account through an adapted version of Loeb's method: a radiative conductivity is calculated in every pore based on its size and shape (Van De Walle and Janssen, 2016). As such, radiation is modelled as a surface-to-surface process in every pore and is incorporated locally 
during the simulation. However, for materials where such modelling formalism is not valid due to thermal radiation transferring partially through the cell walls, the framework can be applied to calculate a conduction-only thermal conductivity $\lambda_{\text {cond,eff }}$, which can be summed with a macroscopic radiative conductivity $\lambda_{\text {rad,eff }}$, as shown in Eq. 2 . This will be further discussed in section 2.3.

$$
\lambda_{\text {eff }}=\lambda_{\text {cond,eff }}+\lambda_{\text {rad,eff }}
$$

The model framework has been validated using a sintered glass filter specimen of $36 \%$ porosity and a Reapor block of $89 \%$ porosity with a mixed cellular-granular pore structure. For both materials, good agreement was achieved between simulation results and experimental measurements, with deviations below 10 \% (Van De Walle et al., 2018). More details on the model framework and the experimental validation can also be found in (Van De Walle, 2019).

To incorporate the effect of nanoscale pores and reduced gas pressures, the next sections discuss their effect on the separate heat transfer mechanisms, together with their implementation in the model framework.

\subsection{Gas-phase conduction}

In high-porosity materials used for building applications, gas-phase conduction typically accounts for the largest part of the total heat transfer through the material. To study the potential strategies for reducing the gas-phase conductivity, insight in the physical process is necessary. Conduction through the gas phase originates from the microscopic movement of gas molecules, transporting their energy over small distances and exchanging energy upon collisions. Several formalisms exist in literature to study the microscopic behaviour and interaction of the gas molecules. In this paper, the kinetic theory of gases framework is used to model the gas-phase conduction and study the impact of reducing gas pressures and pore sizes. Although less accurate than more detailed simulations strategies like the molecular dynamics technique (Bi et al., 2014) or the DSMC technique (Denpoh, 1998; Zhu et al., 2017), it provides reasonably accurate results and an intuitive picture of the effect of reducing the pore size and gas pressure. Furthermore, it allows to extend the existing numerical model in a straightforward way as will be shown further on. First, a short introduction to the kinetic theory of gases is presented, after which the calculation methodology is derived.

\subsubsection{Gas-phase conduction in bulk gases}

The simple kinetic theory of gases framework is an established formalism described for example in the seminal book of Kennard (1938). It allows studying macroscopic properties like the gaseous thermal conductivity by describing the gas as molecules in random motion. It assumes the gas to behave like an ideal gas, meaning gas molecules can be described as spherical masses occupying only a negligible space (dilute) and only interacting through elastic collisions (hence without other intermolecular forces). Such conditions are indeed valid for most gases around room temperature and at atmospheric pressure or below. Each particle is seen as an 'energy carrier', transporting its energy in whichever direction it is flying. When two particles collide, they exchange energy and change the direction of their path. This process will induce energy to flow (on average) from the warm side of the gas to the colder side of the gas.

The framework allows to deduce a formula for the thermal conductivity $\lambda_{\text {gas }}[\mathrm{W} / \mathrm{mK}]$ of such a gas as shown in Eq. 3: 


$$
\lambda_{\text {gas }}=\frac{1}{3} * c_{V, \text { gas }} * \rho_{\text {gas }} * v_{\text {avg,gas }} * L_{M F P}
$$

with, $c_{V, g a s}[\mathrm{~J} / \mathrm{kgK}]$ the specific heat at constant volume, $\rho_{g a s}\left[\mathrm{~kg} / \mathrm{m}^{3}\right]$ the mass density, $v_{\text {avg,gas }}[\mathrm{m} / \mathrm{s}]$ the average speed of the gas molecules and $L_{M F P}[\mathrm{~m}]$ the mean free path of the gas molecules. The specific heat depends on the gas temperature and is tabulated for most gases. The mass density depends on gas pressure $p_{\text {gas }}[\mathrm{Pa}]$ and temperature $T_{\text {gas }}[K]$ and can be calculated using the ideal gas law shown in Eq. 4a, knowing the specific gas constant $R_{\text {specific,gas }}[\mathrm{J} / \mathrm{kgK}]$. The average molecule speed, $v_{\text {avg,gas }}[\mathrm{m} / \mathrm{s}]$, is a representation of the whole speed distribution of the gas molecules. Jiji (2009) proposed to approximate this speed by its root-mean-square value, and hence it depends on the specific gas constant $R_{\text {specific }}$ and the gas temperature $T_{\text {gas }}$ as shown in Eq. $4 b$.

$$
\begin{aligned}
\rho_{\text {gas }} & =\frac{p_{\text {gas }}}{R_{\text {specific,gas }} * T_{\text {gas }}} \\
v_{\text {avg,gas }} & =\sqrt{3 * R_{\text {specific,gas }} * T_{\text {gas }}}
\end{aligned}
$$

Finally, the mean free path $L_{M F P}$ represents the average distance a gas molecule travels between successive collisions. For bulk gases, this depends on the pressure and the temperature of the gas as well as on the gas molecule properties. For example, for air at $20^{\circ} \mathrm{C}$ and atmospheric pressure, the mean free path is about $58 \mathrm{~nm}$.

When applying Eq. 1 with property values found in literature, results consistently underestimate the true gaseous thermal conductivity. As argued by both Zhang and Jiji (Jiji, 2009; Zhang, 2007), this is because the average distance between collisions is the mean free path for momentum transfer, and not for energy transfer. Often, a factor depending on the specific heat ratios $\gamma$ is introduced in Eq. 1 to correct for this issue. However, here we follow the solution proposed by Jiji (2009), defining a 'mean free path for energy exchange' that should be used in Eq. 1 instead of the physical distance between two collisions. This energy-exchange mean free path can be deduced from Eq. 1 using a tabulated value for the gaseous thermal conductivity and specific heat, and using Eqs. 4 for the mass density and average speed. For air at $20{ }^{\circ} \mathrm{C}$ and atmospheric pressure, this leads for example to an energyexchange mean free path of $180 \mathrm{~nm}$, roughly 3 times higher than the physical mean free path.

\subsubsection{Gas-phase conduction at low gas pressures and in pore cavities}

The kinetic theory of gases highlights through Eq. 3 the effect of several parameters on the resulting gaseous thermal conductivity. In this paper, focus is only placed on the effect of the gas pressure and the effect of confinement in a pore cavity, both of which are currently targeted in optimization efforts of thermal insulation materials. These parameters will influence the mean free path of the gas particles and hence the resulting gas-phase conductivity as explained below. A calculation methodology is subsequently derived to predict the gas-phase conductivity in a certain pore depending on its size, shape and gas pressure. 
The effect of the pore size and the gas pressure on the mean free path

When a gas is confined inside a pore cavity, gas molecules will from time to time collide with the pore walls. As such, heat is conducted through the gas from one side of the pore to the other. This affects the actual (or effective) mean free path $L_{M F P, e f f}[\mathrm{~m}]$ of the molecules, which can be estimated with Matthiessen's rule in Eq. 5 assuming independent scattering probabilities for both scattering mechanisms (Jiji, 2009). The importance of such boundary scattering events relative to particleparticle scattering is often indicated through the Knudsen number, defined as the ratio of the bulk gas mean free path to the characteristic length of the system (i.e. the pore size). When the pores are relatively big, the amount of collisions with the wall are relatively small, and the Knudsen number will be low $(<0.1)$. In that case, the effective mean free path almost equals the bulk mean free path $L_{M F P, \text { bulk }}[\mathrm{m}]$. This regime is often called the diffusive regime since heat flow is diffusive from a macroscopic point of view. However, when the pore size reduces to the order of the bulk mean free path, molecules will collide relatively more often with the pore boundaries, and Knudsen number is around 1 . The effective mean free path hence reduces, and so will the gas-phase conductivity. At further reducing pore size (and high Knudsen numbers $>10$ ), gas molecules travel directly from one pore boundary to the other, which is in the limit called the ballistic regime. The mean free path is in that case only influenced by the boundaries and is called the boundary mean free path $L_{M F P, b d y}[\mathrm{~m}]$.

$$
\frac{1}{L_{M F P, \text { eff }}}=\frac{1}{L_{M F P, b u l k}}+\frac{1}{L_{M F P, b d y}}
$$

At reducing gas pressure, on the other hand, the mass density of the gas will decrease according to the ideal gas law since there are fewer molecules per unit volume. At the same time, the bulk mean free path will increase since there are less molecules present to collide with. Both effects counteract each other in Eq. 3, leading to the bulk gaseous thermal conductivity hardly changing with reducing pressure. However, when confined in a pore cavity, the effect of the reducing effective mean free path described above will set in at relatively larger pore sizes. Indeed, since the bulk mean free path of the gas increases at reducing pressure, the effect of the pore confinement becomes already relevant at relatively larger pore sizes.

To calculate the gas-phase conductivity inside a pore, one needs to calculate the effective mean free path and hence the boundary mean free path imposed by the pore boundaries. Expectedly, this boundary mean free path will depend on the pore size and the pore shape. Besides, it also depends on the efficiency of energy exchange between the gas molecule and the pore wall, as will be discussed further on. Similarly to the energy-exchange bulk mean free path, the energy-exchange boundary mean free path should be used in Eq. 5. Except for simple structures like parallel plates, calculating this energy-exchange boundary mean free path is far from trivial. Therefore, a new methodology is proposed here.

\section{Calculating the energy-exchange boundary mean free path}

The energy-exchange boundary mean free path depends on the pore size, pore shape and gasboundary interaction. Accurate calculations of the pore confinement effect so far required the use of complex strategies like molecular dynamics. Here, an intuitive and complete analytical solution is proposed to quantify the energy-exchange boundary mean free path.

The boundary mean free path only relates to the effect a gas molecule experiences from the surrounding boundaries. Thus, by definition it boils down to the situation where no interactions with 
other gas molecules is happening. To calculate this effect, we turn to the analogy with thermal radiation. Indeed, thermal radiation is often modelled as a process between surfaces, without any interaction between the photons. Considering the photons as particles, it hence shows great similarity with the process of gas molecules travelling directly between boundaries without scattering with other gas particles. Furthermore, similar to gas-phase conduction, the kinetic theory framework also allows to formulate an expression for the radiative conductivity as shown in Eq. 6 (Chen, 2005; Jiji, 2009).

$$
\lambda_{\text {rad }}=\frac{1}{3} * C_{\text {rad }} * v_{\text {rad }} * L_{M F P, \text { rad }}
$$

The specific heat $C_{\text {rad }}\left[\mathrm{J} / \mathrm{m}^{3} \mathrm{~K}\right]$ and speed of the photons $v_{\text {rad }}[\mathrm{m} / \mathrm{s}]$ is defined as shown in Eq. 7, with $v_{\text {light }}[\mathrm{m} / \mathrm{s}]$ the speed of light and $\sigma\left[\mathrm{W} / \mathrm{m}^{2} \mathrm{~K}^{4}\right]$ the Stefan-Boltzmann constant.

$$
\begin{gathered}
C_{\text {rad }}=\frac{16 * \sigma * T^{3}}{v_{\text {light }}} \\
v_{\text {rad }}=v_{\text {light }}
\end{gathered}
$$

The effective mean free path for thermal radiation can again be calculated using Matthiessen's rule (Eq. 5). The bulk mean free path of photons is usually several orders of magnitude larger than the cavity size when travelling inside a cavity filled with a non-absorbing medium as air. Hence, for thermal radiation, the effective mean free path equals the boundary mean free path, as shown in Eq. 8.

$$
\frac{1}{L_{M F P, e f f, r a d}}=\frac{1}{L_{M F P, b u l k, r a d}}+\frac{1}{L_{M F P, b d y, r a d}} \cong \frac{1}{L_{M F P, b d y, r a d}}
$$

This boundary mean free path for thermal radiation can be deduced by comparing Eq. 6 with previously derived equations in literature for the radiative thermal conductivity in pores. Based on the methodology of Loeb (Loeb, 1954), the authors presented a model to calculate the radiative conductivity inside a pore cavity as a function of the pore size and shape (Van De Walle and Janssen, 2016). The model was derived based on a large set of detailed radiation simulations performed in pores with varying pore size, shape and orientation. An analytical equation was fitted through the simulation results, allowing to calculate the radiative conductivity in any pore based on its dimensions and shape. The analytical model has been expanded recently to include the effect of the wall emissivity $\epsilon[-]$ and is presented in Eq. 9a-c (Van De Walle, 2019). The pore size is characterized by its mean distance $d_{x}[\mathrm{~m}]$ between the opposing pore walls in the general heat flow direction $x$. The factor $C[-]$ combines the influence of the wall emissivity and the pore slenderness factor $S_{f}[-]$. This slenderness factor characterizes the pore shape and is defined as the ratio between the mean distance between the opposing pore walls in the 3 main directions $\left(d_{x}, d_{y}, d_{z}[m]\right)$. As follows logically from Eq. $9 \mathrm{~b}$, the slenderness factor depends on the general heat flow direction, so possible anisotropic effects can be included. 


$$
\begin{gathered}
\lambda_{\text {rad,pore }}=4 * \sigma * T^{3} * d_{x} * C\left(\epsilon, S_{f}\right) \\
S_{f}=\frac{d_{z} * d_{y}}{d_{x}^{2}} \\
C=\left\{\begin{array}{c}
\left(\frac{0.6615+2.4642 * S_{f}}{0.3526+2.8103 * S_{f}+0.0189 * S_{f}^{2}}\right)^{1.673-3.5092 * \epsilon} *(0.7051 * \epsilon)^{0.9604} \\
\frac{\epsilon}{2-\epsilon}\left(\text { for } S_{f}>8\right)
\end{array}\right.
\end{gathered}
$$

By now equalizing Eq. 6 and 9a, we can deduce an expression for the boundary mean free path for radiation (Eq. 10). Note that this is also an 'energy-exchange' boundary mean free path, since it is not the actual distance a photon travels but is also influenced by the efficiency of energy exchange through the emissivity.

$$
L_{M F P, \text { rad,boundary }}=\frac{3}{4} * d_{x} * C\left(\epsilon, S_{f}\right)
$$

The wave-particle duality of photons allows to interpret all photons as particles flying from surface to surface. This means the pore boundaries have a similar 'confinement' effect on the mean free path travelled by a single photon as they have on a single gas particle (provided there is no collision with other gas particles). Hence, Eq. 10 also applies to the energy-exchange boundary mean free path of gas molecules. Of course, in this equation, the wall emissivity is still present, which is not applicable for gas particles. However, a similar factor characterizes the efficiency of energy transfer between gas molecules and a boundary: the thermal accommodation coefficient $a$ [-]. If $a$ equals 1 , a gas particle hitting the wall will reflect having the same thermal energy as the wall itself, while if its value equals 0 , no energy transfer takes place between the wall and the gas molecules during the collision. For most common materials, this value is around 0.9 , although for very smooth metallic surfaces, the value can be only 0.1 (Zhang, 2007). Due to the physical similarity of this factor with the emissivity, we can replace it in Eq. 10 to obtain finally an expression for the energy-exchange boundary mean free path for gas molecules confined in a pore cavity (Eq. 11).

$$
L_{M F P, \text { gas }, \text { boundary }}=\frac{3}{4} * d_{x} * C\left(a, S_{f}\right)
$$

Combining Eq. 11 with Eq. 3-5, this allows to calculate the gas-phase conductivity in any pore taking into account its size, shape, gas-boundary interaction and pressure. As example, Figure 2 shows the prediction of the thermal conductivity of air confined in a spherical pore for different pore sizes and gas pressures at accommodation coefficient of $0.9(\mathrm{a})$, and for different accommodation coefficients at atmospheric pressure (b). The graphs show clearly the expected decrease of the thermal conductivity with decreasing pore size, gas pressure and/or thermal accommodation coefficient. 

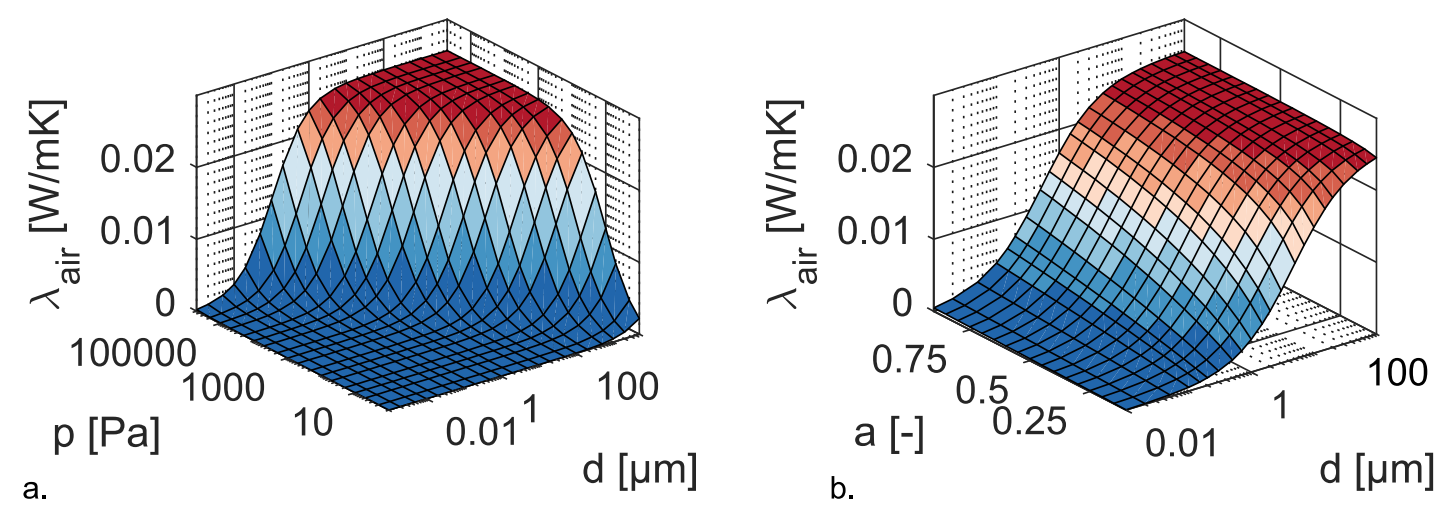

Figure 2: Predicted gaseous thermal conductivity in a spherical pore as a function of the diameter and gas pressure for an accommodation coefficient of $0.9(a)$, and as a function of the diameter and the accommodation coefficient for atmospheric pressure (b).

The presented equations are implemented in the model prediction framework where a separate gaseous thermal conductivity is calculated for every pore based on the pore size, pore shape, gasboundary interaction and gas pressure.

\subsubsection{Validation of gas-phase conductivity calculation}

The previously explained methodology to calculate the gas-phase conduction inside a cavity is validated against results found in literature. First, the gas-phase conductivity between two parallel plates is studied, followed by the gas-phase conductivity inside a cuboid pore.

\section{Gas confined between parallel plates}

Results of two studies are used here to validate the proposed calculation method for the gas-phase conductivity. First, experimental results performed by Braun and Frohn (1976) on the thermal conductivity of Argon gas are studied. The gas was confined between two plates with $1 \mathrm{~mm}$ separation, while the average temperature was kept at $323 \mathrm{~K}$. The gas pressure was varied from 8.5 $\mathrm{Pa}$ to $66735 \mathrm{~Pa}$. The thermal accommodation coefficient of the plates was 0.7385 . On the other hand, the numerical results obtained by Denpoh (1998) with the DSMC technique on the thermal conductivity of $\mathrm{N}_{2}$ gas are used. The gas was kept at $343 \mathrm{~K}$ average temperature and atmospheric pressure. The plate separation distance was varied between $1.6 \mathrm{~nm}$ and $22 \mu \mathrm{m}$. Furthermore, Denpoh explored the effect of different thermal accommodation coefficients by considering three values: 1 , 0.5 and 0.1. Details about both studies are summarized in Table 1.

Table 1: Summary of studies used to validate the gas-phase conductivity calculation methodology.

\begin{tabular}{|c|c|c|c|c|c|c|}
\hline Study & Config. & $\mathrm{Gas}$ & $\mathrm{T}[\mathrm{K}]$ & $\mathrm{p}[\mathrm{Pa}]$ & Size $[\mathrm{m}]$ & $\mathrm{a}[-]$ \\
\hline $\begin{array}{c}\text { Braun \& Frohn } \\
1976\end{array}$ & $/ /$ plates & $\mathrm{Ar}$ & 323 & $8.5-66735$ & $1 * 10^{-3}$ & 0.7385 \\
\hline $\begin{array}{c}\text { Denpoh } \\
1998\end{array}$ & $/ /$ plates & $\mathrm{N} 2$ & 343 & 101325 & $\begin{array}{l}1.6 * 10^{-9}- \\
22 * 10^{-6}\end{array}$ & $0.1 / 0.5 / 1$ \\
\hline $\begin{array}{c}\text { Zhu et al. } \\
2017\end{array}$ & Cuboid & $\mathrm{Ar}$ & 300 & 101000 & $\begin{array}{l}24 * 10^{-9}- \\
1.4 * 10^{-6}\end{array}$ & 1 \\
\hline
\end{tabular}

Using the proposed calculation method (Eq. 3-5 \& 11), the gas-phase conductivities in both presented studies are predicted. Necessary properties of the bulk gas $\left(c_{V, \text { gas }}, \lambda_{\text {bulk,gas }} \& R_{\text {specific }}\right)$ are found in specialized literature (Engineering ToolBox, 2004; NIST, 2018). The obtained results are compared with the results of both studies in Figure 3a. Results are shown as a function of the plate separation distance 
normalized by the collision mean free path of the respective gas. As can be seen, results agree very well with the literature results, both for the case of a reducing gas pressure (Braun \& Frohn) and a reducing plate separation distance (Denpoh). Furthermore, the effect of the thermal accommodation coefficient is very well predicted using the energy-exchange boundary mean free path calculation. Also shown are the results obtained with the often applied analytical formula for gaseous heat conduction between parallel plates, described for example in the book by Zhang (2007). As expected, results show again a very good agreement.

Gas in a cuboid nanopore

Zhu et al. (2017) numerically studied the gas-phase conductivity of Argon gas confined in a cuboid pore using the DSMC technique. The gas was set to atmospheric pressure and a temperature of 300 $\mathrm{K}$, the thermal accommodation coefficient to 1 . The pore's side length was varied between $24 \mathrm{~nm}$ and $1.4 \mu \mathrm{m}$. Details are again summarized in Table 1. The proposed methodology is again applied to calculate the gas-phase conductivities for the cases presented in the study by Zhu et al. Results are shown in Figure 3b. Again, a very good agreement is obtained between the numerical results obtained through the proposed model and the DSMC technique. Also shown are the results obtained using the analytical expression for gas-phase conduction between parallel plates (Zhang, 2007). This time, less agreement is obtained with the numerical results. This is due to the cuboid shape of the pore influencing the gas-phase conductivity, while the analytical expression was derived for parallel plates. This highlights the importance of taking into account the pore shape, as is done in the newly proposed methodology.
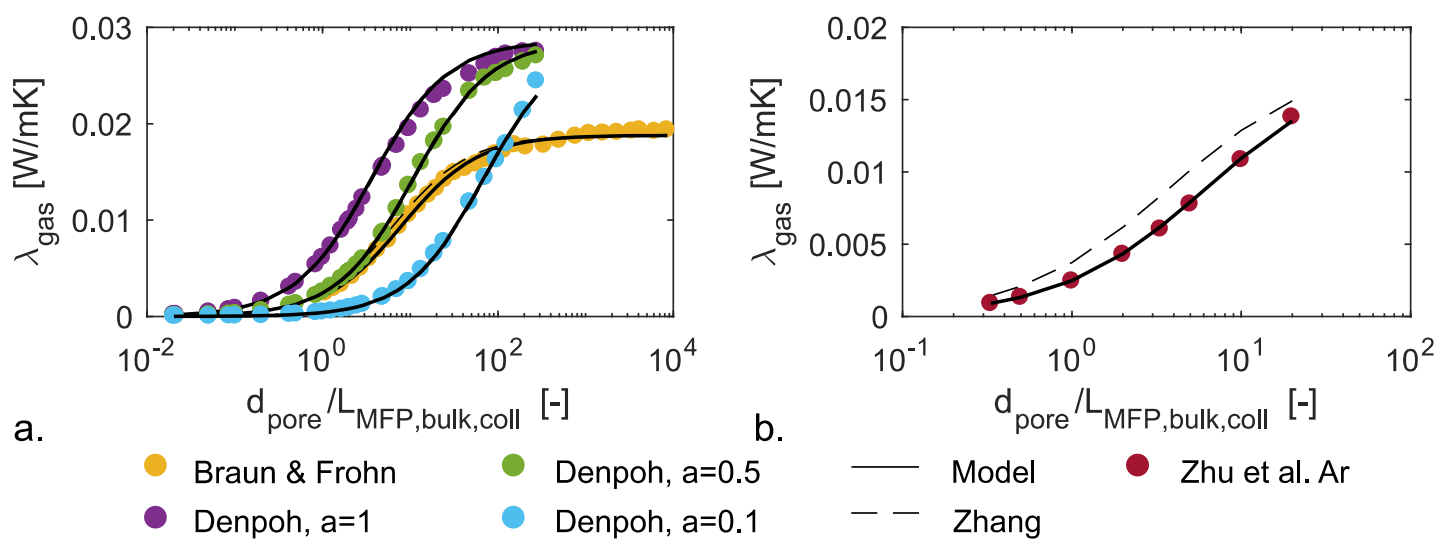

Figure 3: Gas-phase conductivity results predicted by the new model agree very well with experimental and numerical results in literature, both for gas confined between parallel plates (a), and gas confined in a cuboid pore (b).

\subsection{Thermal radiation}

Radiative heat transfer in porous materials can contribute significantly to the total heat transfer through the material, certainly at high porosities and high temperatures. For macro-porous cellular materials, the authors proposed to model thermal radiation as a process from pore wall to pore wall, following the methodology of Loeb and other authors (Bakker et al., 1995; Loeb, 1954; Van De Walle and Janssen, 2016). As such, it is assumed that all radiation is emitted and absorbed at the pore surface. However, with reducing pore size, the pore cell wall thickness will be reduced as well. Below a certain thickness, thermal radiation will transfer partially through the cell wall (Glicksman and Torpey, 1989). Thus, the surface-to-surface model is expected to be not valid for nano-porous cellular materials and might underestimate the contribution of thermal radiation to the total heat transfer. Since radiation waves can travel through several pores and pore walls before being extinct, it makes more sense to model the process on a material scale, taking into account as much as possible the local 
structural properties of the pore structure (i.e. cell wall thickness, pore size, refractive index of material).

Detailed modelling of the full interaction between the radiation waves and the complete pore structure is fairly complex. Some very detailed studies have been performed in literature on several types of foams, using for example ray-tracing algorithms (Baillis et al., 2013; Coquard et al., 2013; Petrasch et al., 2007). Such simulation strategies allow to carefully study the impact of very specific pore scale parameters like cell size, strut geometry or wall thickness. However, due to their computational intensiveness, in this study an analytical approach proposed by Wang et al. (Wang et al., 2018; Wang et al., 2017a) is adopted which shows very good agreement with experimentally measured data.

A macroscopic radiative conductivity is calculated for the whole material and summed with the conduction-only conductivity of the material (Eq. 12). The conduction-only conductivity is obtained in this paper with the detailed image-based model explained before.

$$
\lambda_{\text {eff }}=\lambda_{\text {cond,eff }}+\lambda_{\text {rad,eff }}
$$

Wang et al. (2017a) proposed to calculate the macroscopic radiative conductivity similar to the approach proposed by De Micco \& Aldao (2003). It hence depends on the average temperature $T_{\text {avg }}[K]$, the cell size $d_{\text {pore }}[\mathrm{m}]$, cell wall thickness $d_{\text {wall }}[\mathrm{m}]$, the sample thickness $d_{\text {sample }}[\mathrm{m}]$ and the net transmittance through 1 unit cell $T_{\text {net }}^{\text {unit }}[-]$ (Eq. 13).

$$
\lambda_{\text {rad,macro }}=\frac{4 * \sigma * T_{\text {avg }}^{3} * d_{\text {sample }}}{1+\frac{d_{\text {sample }}}{d_{\text {pore }}+d_{\text {wall }}} *\left(\frac{1}{T_{\text {net }}^{\text {unit }}}-1\right)}
$$

To come to an analytical expression for the net transmittance of radiation through a unit cell, Wang et al. simplified the pore structure as a set of cubic cells made by orthogonal plates representing the matrix (Figure 4). Thermal radiation will interact with the cell walls by reflection, transmission and absorption. Since the roughness of the cell walls is relatively small compared to the wavelength of the thermal radiation $(\sim 10 \mu \mathrm{m})$, all reflection is assumed to be specular. The matrix walls that are parallel to the direction of general heat flow will hence only contribute to the attenuation of the radiation by absorption, since the specular reflection will not change the component of the wave's direction parallel to the general heat transfer direction. The cell walls perpendicular to the heat flow will attenuate the radiation both by absorption and reflection. Hence, Wang et al. simplified such a unit cell as three pore walls parallel to each other, all three absorbing equally the thermal radiation while only the first reflects thermal radiation (Figure 4). 


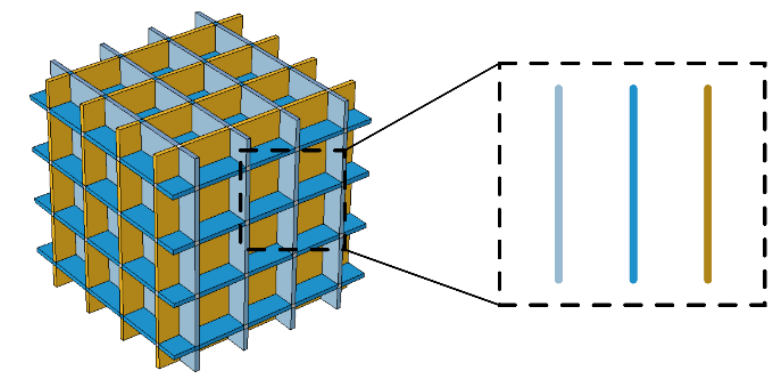

Figure 4: Simplified pore structure representation used to derive the analytical model for thermal radiation by Wang et al. (2017a).

The absorbance, reflectance and transmittance of one cell wall are calculated using the expressions formulated by Born \& Wolf for each wavelength, angle of incidence and polarization (Born and Wolf, 2003). The type of material constituting the cell walls influences these properties through the material's complex refractive index $n+i \kappa$. The thin film interference effect, occurring due to reflection of thermal radiation on both interfaces of the cell wall, is naturally incorporated in these formulas. The properties are averaged for both polarizations, weight-averaged with the wavelengthdependent emission density and integrated over all incident directions to obtain the total absorbance, reflectance and transmittance of a cell wall. It is assumed that half of the absorbed radiation is reemitted forwards while the other half is re-emitted backwards. The net fraction of radiation transmitted through the unit cell can then be calculated taking into account multiple reflections, absorption and re-emission of the cell walls.

Wang et al. (2017a) validated the analytical calculation of the thermal radiation with literature data on XPS foams. Very good agreement was obtained in predicting the radiative component, with relative deviations below $10 \%$.

\subsection{Solid conduction}

For non-electrically-conducting solids, matrix conduction occurs due to the vibration of atoms. The quantized vibration waves are usually called phonons. For crystalline materials, the kinetic theory of gases framework is often applied to estimate the effect of reducing material thickness on the material's thermal conductivity, considering the phonons as a phonon gas. For amorphous materials, like polymers or glass, the mechanism of solid conduction is much less understood (Kommandur and Yee, 2017; Wingert et al., 2016). The analogy of a phonon gas has been shown to be less applicable here, and the accuracy of the kinetic theory framework approximation becomes hence highly questionable for the intended application of the presented model (Cahill and Pohl, 1988; Lv and Henry, 2016). A proper interpretation of the matrix conduction requires atomistic simulation strategies, which is outside the scope of this paper. Nonetheless, the kinetic framework has been applied previously in literature to obtain a first idea of the effect of the cell wall size on the matrix conductivity, as for example by $\mathrm{Hu}, \mathrm{Wu} \&$ Sun or Wang et al. (Hu et al., 2018; Wang et al., 2017a). They showed that, using such analogy, the mean free path of the phonons in typical low-conducting polymers used to create insulation materials is on the order of 1-2 $\mathrm{nm}$. Hence, the reducing effect on the solid thermal conductivity only sets in at cell wall thicknesses below $10 \mathrm{~nm}$. This is also confirmed by results with molecular dynamics on thin polystyrene films ( $M a$ and Tian, 2015). Given that such small wall thicknesses are not considered relevant for our study, in this paper the matrix conductivity is considered as an a priori known parameter and kept fixed during the simulation. 


\section{Model framework validation}

The previous section discussed the implementation of the different heat transfer mechanisms in the model framework, taking into account reduced pore sizes and gas pressures. The gas-phase conduction implementation was furthermore validated with data found in literature. This section discusses the performance of the model for predicting the thermal conductivity of a nanoporous material, where all three heat transfer mechanisms occur at the same time. The performance of the model for macroporous materials was already validated in earlier work by the authors (Van De Walle et al., 2018).

\subsection{Material}

The model framework is applied to predict the thermal conductivity of nanocellular foams. Similar to better known and often used microcellular foams like PUR and XPS, these foams are made of a polymer matrix containing pores with nanoscale dimensions. Although currently only producible in small amounts and small dimensions, several literature papers discuss the potential improvements of these foams regarding thermal and structural properties (Costeux, 2014). In this paper, the model framework is compared to experimental measurements performed by Wang et al. (2017b) on PMMA based foams. Varying the foaming process parameters, four foams were created with average pore sizes between $205 \mathrm{~nm}$ and $4.63 \mu \mathrm{m}$ and porosities between 86 and $91 \%$, as summarized in Table 2 . All foams have a mono-modal pore size distribution, but no further information on the distribution width is detailed. Their thermal conductivity, measured with a Hot Disk TPS 2500 sensor, is also summarized in Table 2. As can be seen, the reduced pore size has a reduction effect on the foam's effective conductivity.

Table 2: Summary of the experimental results of four nanocellular foams produced and characterized by Wang et al. (2017b)

\begin{tabular}{|c|c|c|}
\hline Pore size [nm] & Porosity [\%] & ETC [W/mK] \\
\hline 205 & 87.5 & 0.0248 \\
\hline 930 & 86.1 & 0.0369 \\
\hline 2860 & 90.4 & 0.0362 \\
\hline 4630 & 88.7 & 0.0383 \\
\hline
\end{tabular}

\subsection{Simulation setup}

The model framework extended in this paper is applied to predict the ETC of the foamed specimens presented by Wang et al. Given that no actual $\mu \mathrm{CT}$ images are available, a virtual generation algorithm is employed to create a foam-like microstructure mimicking the material's true microstructure. The generation algorithm, as described by Randrianalisoa et al. (2013), is based on the Voronoï technique. First, a number of seeding points are located on a body-centred cubic lattice in a cubic volume, and slightly displaced in a random direction according to a perturbation factor. The Voronoï technique will divide the volume in separate cells based on the seeding points. The thickness of the cell walls is varied to obtain different porosities for the same pore locations. A strut-rounding algorithm based on dilation and erosion with a spherical element was implemented to obtain more realistic microstructures. A SEM picture by Wang et al. (2017b) of a foam with an average pore size of $205 \mathrm{~nm}$ is shown in Figure 5 a. An example of a generated foam-like sample is shown in Figure $5 b-c$ together with a cross-section through the middle. The generated foam shows good visual agreement with the SEM picture. 


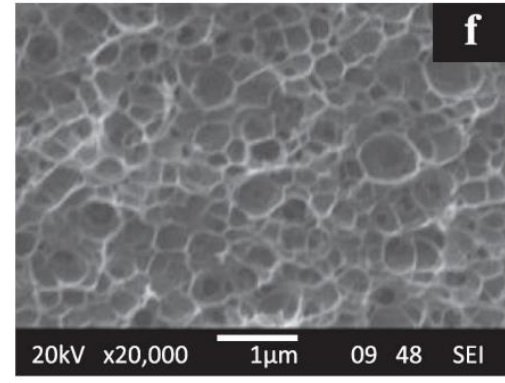

a.

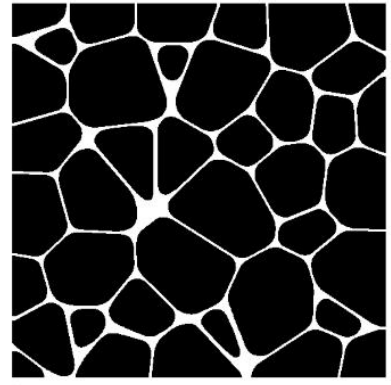

b.

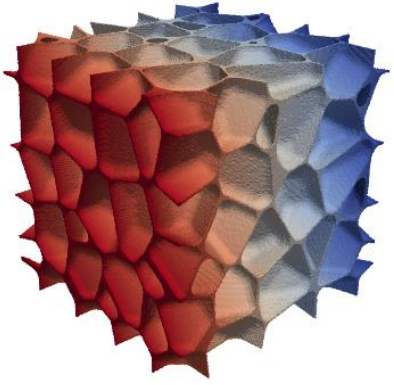

c.

Figure 5: SEM picture of a foam specimen by Wang et al. (2017b) (a), slice of a virtually generated sample (b) and 3D view of a virtually generated sample (c).

Three different pore structures are generated with a perturbation factor of 0.3 , resulting in a good visual agreement between the virtually generated samples and the SEM pictures of the samples given in Wang et al. (2017b), see Figure 5a\&b. Exploratory simulations with different perturbation factors indicated only a secondary impact for the considered material. The cell wall thickness of each sample is adapted to obtain three different porosities for each pore structure. Subsequently, the size of the voxels is rescaled to obtain samples with four different average pore sizes similar to the experimentally created samples. Each sample of the resulting 36 samples is simulated with the model framework explained and expanded in the paper. The thermal conductivity of the solid PMMA matrix was set to $0.2098 \mathrm{~W} / \mathrm{mK}$, consistent to the value measured by Wang et al. (2017b). The properties of the air inside the pores are found in literature or calculated using Eq. 4 and are summarized in Table 3. A thermal accommodation coefficient of 0.9 was assumed for the air-PMMA interface, a value commonly assumed in literature (Zhang, 2007). The gas temperature and pressure are taken as $23^{\circ} \mathrm{C}$ and $101325 \mathrm{~Pa}$, similar to the conditions reported by Wang et al (2017b). Thermal simulations are performed on a machine with 60 cores, yielding results in approximately 30 minutes.

Table 3: Thermophysical properties of air at $23^{\circ} \mathrm{C}$ and atmospheric pressure.

\begin{tabular}{|l|l|}
\hline Property & Value \\
\hline$R_{\text {Specific }}$ (Engineering ToolBox, 2004) & $287 \mathrm{~J} / \mathrm{kgK}$ \\
\hline$\rho_{\text {air }}$ (Eq. 4) & $1.19 \mathrm{~kg} / \mathrm{m}^{3}$ \\
\hline$c_{V}$ (NIST, 2018) & $717 \mathrm{~J} / \mathrm{kgK}$ \\
\hline$v_{\text {avg,gas }}$ (Eq. 4) & $505 \mathrm{~m} / \mathrm{s}$ \\
\hline$\lambda_{\text {gas,bulk }}$ (NIST, 2018) & $0.0259 \mathrm{~W} / \mathrm{mK}$ \\
\hline
\end{tabular}

For the radiative part, the model by Wang et al. (2017a) is used as explained previously. The complex refractive index of the PMMA matrix is approximated as 1.47+0.02i for the most pertinent wavelength of $10 \mu \mathrm{m}$ of room temperature thermal radiation (Berisha et al., 2016). The cell wall thickness is deduced from the 3D image of the generated samples by a distance transformation algorithm and is weight-averaged by the cell wall area. The material thickness is taken as $1 \mathrm{~cm}$, comparable to the dimensions of the measured foam samples. The total effective conductivity is finally obtained by summing the conductive part obtained using the 3D model with the radiative part obtained using the analytical model.

\subsection{Results \& Discussion}

The conduction-only results obtained using the 3D model for the 36 simulated samples are compared with the four experimental results in Figure 6a. Results are presented as function of the samples' porosity while the color highlights the average pore size of the sample. A least-squares power-law 
trendline is fitted through the simulations for every pore size. The error bars on the experimental measurements present the $+/-5 \%$ uncertainty associated with the Hot Disk sensor. As can be seen, the conduction-only results agree already very well with all four measurements. Deviations with the per-pore-size simulation trendlines are below $10 \%$ at the corresponding porosity, for all pore sizes. Simulation results furthermore confirm the expected decrease of the thermal conductivity with increasing porosity and/or decreasing pore size. A decrease of over $40 \%$ when reducing the average pore size from $4.6 \mu \mathrm{m}$ to $205 \mathrm{~nm}$ is found at these relatively high porosities.

Simulation results for the radiative conductivity are shown in Figure $6 \mathrm{~b}$. They show an increasing trend with porosity due to the decreasing amount of absorbing material in the samples. Furthermore, they also highlight an increasing trend with decreasing pore size, contrary to the conduction-only results. This is explained by the decreasing cell wall thickness for decreasing pore sizes. The effect of the increasing transmissivity of such thin cell walls outweighs the counteracting effect of an increasing number of cell walls per meter of sample thickness, as was already argued by Wang et al. (2017a). The absolute values of the radiative conductivity are relatively small, due to the high absorption coefficient of the PMMA material constituting the matrix.
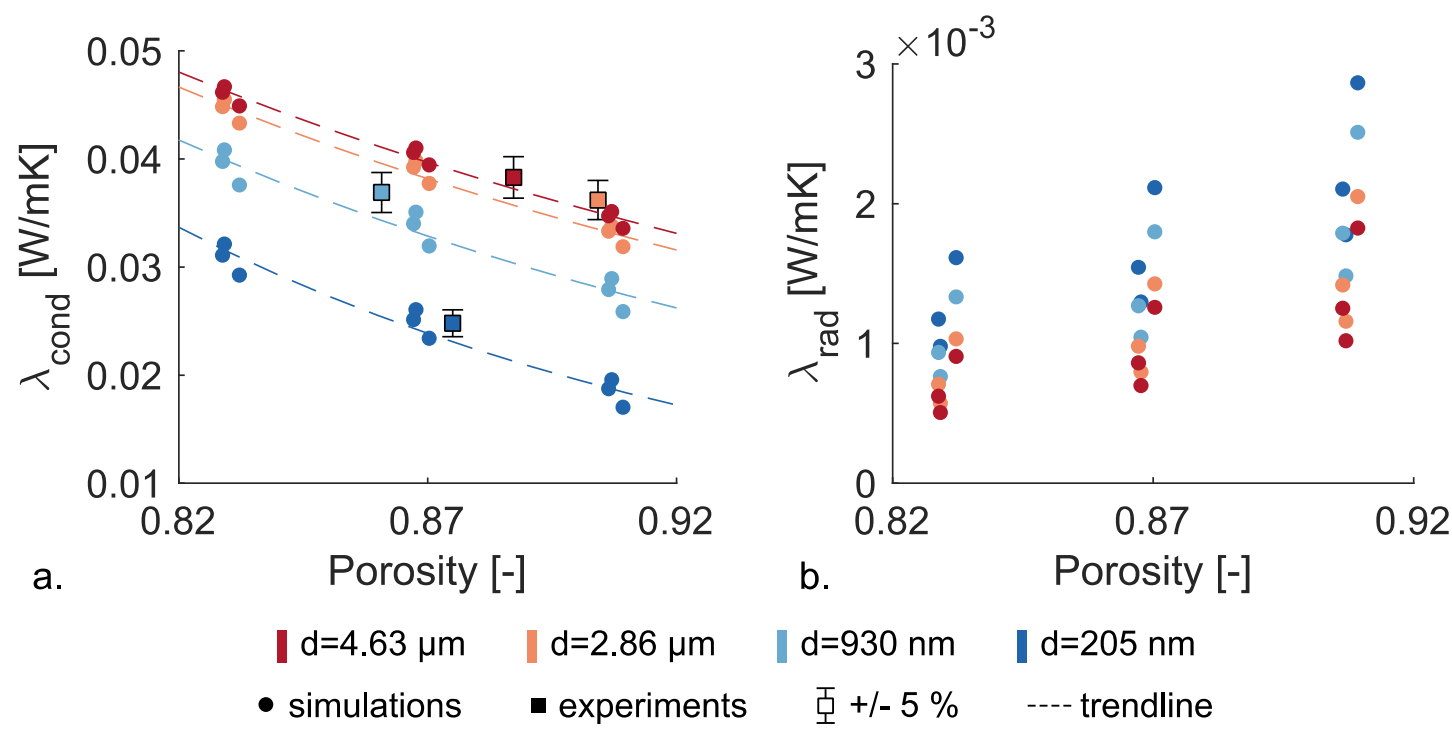

Figure 6: Comparison of simulated conduction-only conductivity with experimental results (a); results obtained for radiative conductivity $(b)$.

The results for the combined effective conductivity of the simulated samples are shown in Figure 7. As can be seen, including the thermal radiation improves the agreement between the simulation results and the experimental results. Only a $5 \%$ difference occurs at the respective porosity with the corresponding simulation trendlines. This indicates the well-performance of the model and its capability of predicting the effect of decreasing the average pore size to nanoscale dimensions. Since the implemented physical description of the gas-phase conduction in the model functions the same for low-pressure samples, it is expected that equally good results will also be obtained for lowpressure materials.

The simulation and experimental results also allow to assess the potential improvement of reducing the pore size. Indeed, a reduction of about $30 \%$ is found when reducing the average pore size from $4.6 \mu \mathrm{m}$ to $205 \mathrm{~nm}$ at $83 \%$ porosity, mounting even to $40 \%$ reduction at $91 \%$ porosity. For the studied material at $83 \%$ porosity, decreasing the pore size seems to have almost the same effect as increasing the porosity with $10 \%$. Of course, in low-porosity materials $(<65 \%)$, the effect of a reducing pore size 
will be less pronounced, since gas-phase conduction is less pertinent in that case. Furthermore, for materials with a less absorbing solid matrix, thermal radiation will play a more important role at increasing porosities and decreasing pore sizes. Indeed, for the studied material, radiative heat transfer contributed only $2-15 \%$ to the total thermal conductivity, depending on the pore size, porosity and wall thickness. In future work, the model framework will be employed to study the impact of the mentioned structural and physical parameters.

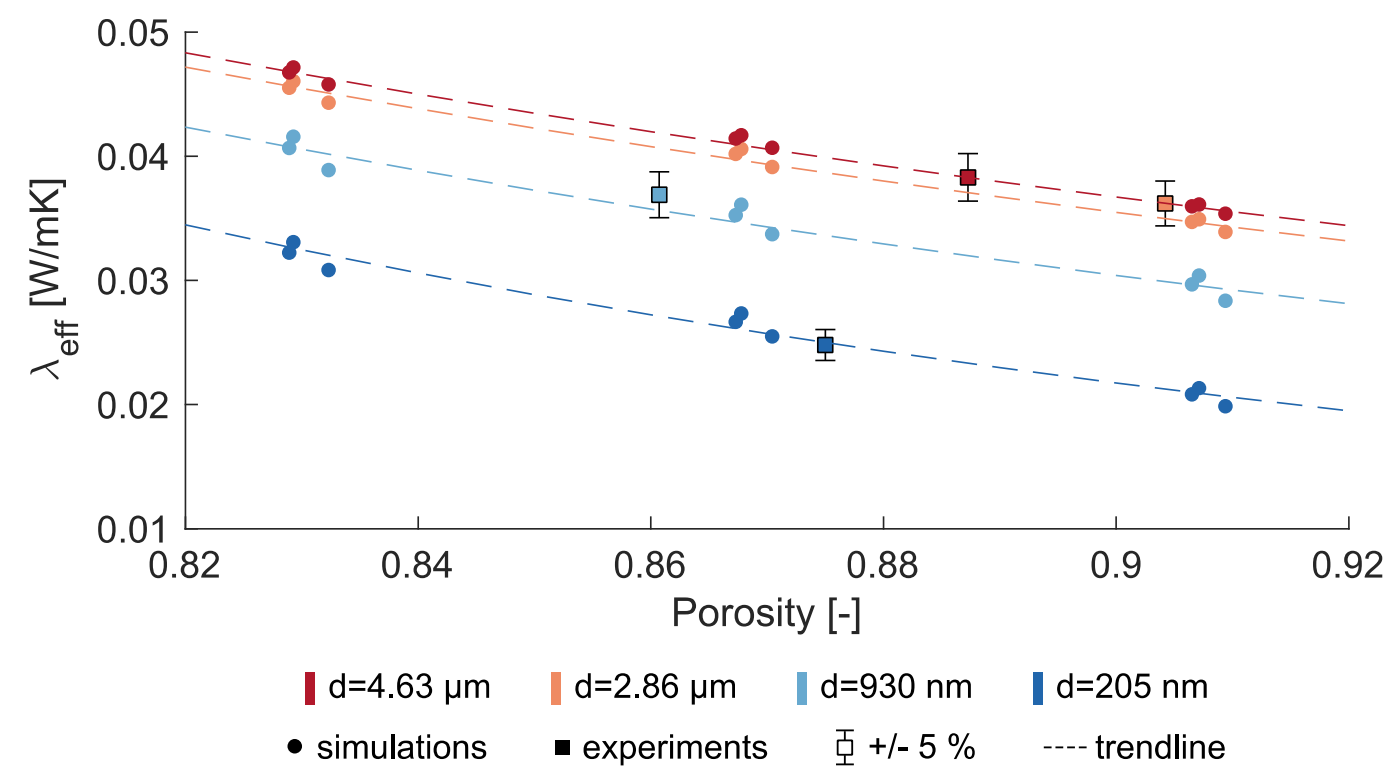

Figure 7: Comparison of simulated effective conductivity with experimental results show very good agreement for all porosities and average pore sizes $\left(T_{\text {avg }}=23^{\circ} \mathrm{C} \& p_{\text {gas }}=101325 \mathrm{~Pa}\right)$.

\section{Conclusions}

Improving the thermal conductivity of porous building materials is one of the key pathways to decrease the heating needs of the building industry. Reducing the pore size or the gas pressure in porous materials can lead to significant improvements, as is the case in novel materials like vacuum insulation and aerogel materials. This paper discussed the extension of a 3D image-based model framework to quantify the effect of such parameters on the effective thermal conductivity of cellular materials. A simple calculation method was developed based on the analogy with thermal radiation, to quantify the gas-phase conductivity in a pore based on its local dimensions, shape, gas-wall interaction and gas pressure. Validation of the method with experimental results from literature showed very good agreement for predicting the gas-phase thermal conductivity at different gas pressures and pore sizes. The gas-phase conductivity calculation has been implemented in the larger model framework to study the ETC of cellular porous materials based on their 3D pore structure. Together with an analytical method to account for thermal radiation, the framework was applied to predict the thermal conductivity of four nanocellular foam specimens experimentally measured in literature. Simulation results agreed within $5 \%$ difference of the measured thermal conductivities for all pore sizes and porosities. Furthermore, they also indicated a potential reduction of the effective thermal conductivity with about $40 \%$ when reducing the pore size from a few micrometre to a few 100 nanometre for such high- porosity low-matrix-conductivity materials. The model framework can thus help in exploring new opportunities for designing materials with specific properties. 


\section{Acknowledgements}

Research funded by a Ph.D. grant of the Agency for Innovation by Science and Technology (IWT-Vlaio). The computational resources and services used in this work were provided by the VSC (Flemish Supercomputer Center), funded by the Research Foundation - Flanders (FWO) and the Flemish Government - department EWI

\section{References}

Alliez P, Jamin C, Rineau L, et al. (2015) 3D Mesh Generation. In: Board CE (ed.) CGAL User and Reference Manual. 4.5. CGAL Editorial Board.

Ashby MF and Medalist RFM (1983) The mechanical properties of cellular solids. Metallurgical Transactions A 14(9): 1755-1769.

Baetens R, Jelle BP, Thue JV, et al. (2010) Vacuum insulation panels for building applications: A review and beyond. Energy and Buildings 42(2): 147-172. DOI: 10.1016/j.enbuild.2009.09.005.

Baillis D, Coquard R, Randrianalisoa J, et al. (2013) Thermal Radiation Properties of Highly Porous Cellular Foams. Special Topics \& Reviews in Porous Media - An International Journal 4(2): 111136. DOI: 10.1615/SpecialTopicsRevPorousMedia.v4.i2.20.

Bakker K, Kwast $\mathrm{H}$ and Cordfunke EHP (1995) The contribution of thermal radiation to the thermal conductivity of porous UO2. Journal of Nuclear Materials 223: 135-142.

Bao H, Chen J, Gu X, et al. (2018) A review of Simulation Methods in Micro/Nanoscale Heat Conduction. ES Energy \& Environment. DOI: 10.30919/esee8c149.

Berisha S, van Dijk T, Bhargava R, et al. (2016) BIM-Sim: Interactive Simulation of Broadband Imaging Using Mie Theory. Microscopy and Microanalysis 22(S3): 1438-1439. DOI: 10.1017/S1431927616008035.

Bi C, Tang G, Sheng Q, et al. (2014) Study on Thermal Conductivity of Gas Phase in Nano-porous Aerogel. (September): 7-10.

Born M and Wolf E (2003) Principles of Optics. 7th expand. Cambridge: Cambridge University Press.

Braun D and Frohn A (1976) Heat transfer in simple monatomic gases and in binary mixtures of monatomic gases. International Journal of Heat and Mass Transfer 19(11): 1329-1335. DOI: 10.1016/0017-9310(76)90085-5.

Cahill DG and Pohl RO (1988) Lattice Vibrations And Heat Transport In Crystals And Glasses. Annual Review of Physical Chemistry 39(2): 93-121. DOI: 10.1146/annurev.physchem.39.1.93.

Chen G (2005) Nanoscale Energy Transport and Conversion: A Parallel Treatment of Electrons, Molecules, Phonons and Photons. New York: Oxford University Press.

Chen J, Zhang M, Wang $\mathrm{H}$, et al. (2015) Evaluation of thermal conductivity of asphalt concrete with heterogeneous microstructure. Applied Thermal Engineering 84. Elsevier Ltd: 368-374. DOI: 10.1016/j.applthermaleng.2015.03.070.

Coquard R, Randrianalisoa J and Baillis D (2013) Computational prediction of radiative properties of polymer closed-cell foams with random structure. Journal of Porous Media 16(2): 137-154. 
Coquil T, Fang J and Pilon L (2011) Molecular dynamics study of the thermal conductivity of amorphous nanoporous silica. International Journal of Heat and Mass Transfer 54(21-22). Elsevier Ltd: 45404548. DOI: 10.1016/j.ijheatmasstransfer.2011.06.024.

Costeux S (2014) CO2-blown nanocellular foams. Journal of Applied Polymer Science 131(23): 41293. DOI: 10.1002/app.41293.

Cuce E, Cuce PM, Wood CJ, et al. (2014) Toward aerogel based thermal superinsulation in buildings: A comprehensive review. Renewable and Sustainable Energy Reviews 34: 273-299. DOI: 10.1016/j.rser.2014.03.017.

De Micco C and Aldao CM (2003) On the net radiation method for heat transfer. European Journal of Physics 24: 81-89.

Denpoh K (1998) Modeling of rarefied gas heat conduction between wafer and susceptor. IEEE Transactions on Semiconductor Manufacturing 11(1): 25-29. DOI: 10.1109/66.661281.

Engineering ToolBox (2004) Universal and Individual Gas Constants. Available at: https://www.engineeringtoolbox.com/individual-universal-gas-constant-d_588.html (accessed 15 June 2018).

Fang $Q$ and Boas DA (2009) Tetrahedral mesh generation from volumetric binary and gray scale images. In: Proceedings IEEE International Symposium on Biomedical Imaging, 2009, pp. 11421145.

Ferkl P, Pokorný R, Bobák M, et al. (2013) Heat transfer in one-dimensional micro- and nano-cellular foams. Chemical Engineering Science 97. Elsevier: 50-58. DOI: 10.1016/j.ces.2013.04.018.

Gibson LJ and Ashby MF (1997) Cellular Solids: Structure and Properties. 2nd ed. Cabridge University Press.

Glicksman LR and Torpey M (1989) Factors Governing Heat Transfer Through Closed Cell Foam Insulation. Journal of Thermal Insulation 12: 257-269.

Guo Y and Wang M (2015) Phonon hydrodynamics and its applications in nanoscale heat transport. Physics Reports 595. Elsevier B.V.: 1-44. DOI: 10.1016/j.physrep.2015.07.003.

He Y-L and Xie T (2015) Advances of thermal conductivity models of nanoscale silica aerogel insulation material. Applied Thermal Engineering. Elsevier Ltd. DOI: 10.1016/j.applthermaleng.2015.02.013.

Hu F, Wu S and Sun Y (2018) Hollow-Structured Materials for Thermal Insulation. Advanced Materials 1801001: 1-17. DOI: 10.1002/adma.201801001.

Jelle BP (2011) Traditional, state-of-the-art and future thermal building insulation materials and solutions - Properties, requirements and possibilities. Energy and Buildings 43(10): 2549-2563. DOI: 10.1016/j.enbuild.2011.05.015.

Jelle BP, Gustavsen A and Baetens R (2010) The path to the high performance thermal building insulation materials and solutions of tomorrow. Journal of Building Physics 34(2): 99-123. DOI: 10.1177/1744259110372782.

Jiji LM (2009) Heat Conduction. 3rd ed. 
Kan A, Kang L, Wang C, et al. (2015) A simple and effective model for prediction of effective thermal conductivity of vacuum insulation panels. Future Cities and Environment 1(1). Future Cities and Environment: 4. DOI: 10.1186/s40984-015-0001-z.

Kennard EH (1938) Kinetic Theory of Gases. 1st ed. New York: McGraw-Hill.

Kommandur S and Yee SK (2017) An empirical model to predict temperature-dependent thermal conductivity of amorphous polymers. Journal of Polymer Science, Part B: Polymer Physics 55(15): 1160-1170. DOI: 10.1002/polb.24365.

Liu S, Duvigneau J and Vancso GJ (2015) Nanocellular polymer foams as promising high performance thermal insulation materials. European Polymer Journal 65. Elsevier Ltd: 33-45. DOI: 10.1016/j.eurpolymj.2015.01.039.

Loeb A (1954) Thermal Conductivity: VIII, A theory of thermal conductivity of porous materials. Journal of the American Ceramic Society 37(2): 96-99.

Lv W and Henry A (2016) Examining the Validity of the Phonon Gas Model in Amorphous Materials. Scientific Reports 6. Nature Publishing Group: 1-8. DOI: 10.1038/srep37675.

Ma H and Tian Z (2015) Effects of polymer chain confinement on thermal conductivity of ultrathin amorphous polystyrene films. Applied Physics Letters 107(7). DOI: 10.1063/1.4929426.

Machrafi $\mathrm{H}$ and Lebon $\mathrm{G}$ (2015) Size and porosity effects on thermal conductivity of nanoporous material with an extension to nanoporous particles embedded in a host matrix. Physics Letters, Section A: General, Atomic and Solid State Physics 379(12-13). Elsevier B.V.: 968-973. DOI: 10.1016/j.physleta.2015.01.027.

NIST (2018) NIST Chemistry WebBook SRD 69 - Thermophysical properties of fluid systems. Available at: https://webbook.nist.gov/chemistry/fluid/ (accessed 15 June 2018).

Petrasch J, Wyss P and Steinfeld A (2007) Tomography-based Monte Carlo determination of radiative properties of reticulate porous ceramics. Journal of Quantitative Spectroscopy and Radiative Transfer 105(2): 180-197. DOI: 10.1016/j.jqsrt.2006.11.002.

Randrianalisoa J, Coquard R and Baillis D (2013) Microscale direct calculation of solid phase conductivity of voronoi's foams. Journal of Porous Media 16(5): 411-426. DOI: 10.1615/JPorMedia.v16.i5.30.

Schiavoni S, D'Alessandro F, Bianchi F, et al. (2016) Insulation materials for the building sector: A review and comparative analysis. Renewable and Sustainable Energy Reviews 62: 988-1011. DOI: 10.1016/j.rser.2016.05.045.

Smith DS, Alzina A, Bourret J, et al. (2013) Thermal conductivity of porous materials. Journal of Materials Research 28(17): 2260-2272. DOI: 10.1557/jmr.2013.179.

Solórzano E, Rodriguez-Perez MA, Lázaro J, et al. (2009) Influence of Solid Phase Conductivity and Cellular Structure on the Heat Transfer Mechanisms of Cellular Materials: Diverse Case Studies. Advanced Engineering Materials 11(10): 818-824. DOI: 10.1002/adem.200900138.

Van De Walle W (2019) Prediction and optimization of the effective thermal conductivity of porous building blocks based on their pore structure. KU Leuven.

Van De Walle W and Janssen H (2016) A thermal conductivity prediction model for porous building 
blocks. Bauphysik 38(6): 340-347. DOI: 10.1002/bapi.201610037.

Van De Walle W, Claes S and Janssen H (2018) Implementation and validation of a 3D image-based prediction model for the thermal conductivity of cellular and granular porous building blocks. Construction and Building Materials 182. Elsevier Ltd: 427-440. DOI: 10.1016/j.conbuildmat.2018.06.105.

Wang Guilong, Wang C, Zhao J, et al. (2017) Modelling of thermal transport through a nanocellular polymer foam: toward the generation of a new superinsulating material. Nanoscale 9(18). Royal Society of Chemistry: 5996-6009. DOI: 10.1039/C7NR00327G.

Wang Guilong, Zhao J, Mark LH, et al. (2017) Ultra-tough and super thermal-insulation nanocellular PMMA/TPU. Chemical Engineering Journal 325. Elsevier B.V.: 632-646. DOI: 10.1016/j.cej.2017.05.116.

Wang Guilong, Wang C, Zhao J, et al. (2018) Correction: Modelling of thermal transport through a nanocellular polymer foam: Toward the generation of a new superinsulating material. Nanoscale 10(43). Royal Society of Chemistry: 20469-20473. DOI: 10.1039/c7nr00327g.

Wingert MC, Zheng J, Kwon S, et al. (2016) Thermal transport in amorphous materials : a review. IOP Publishing.

Zhang ZM (2007) Nano/Microscale Heat Transfer. The McGraw-Hill Companies, Inc. DOI: 10.1007/s10765-008-0380-6.

Zhu C-Y, Li Z-Y and Tao W-Q (2017) Theoretical and DSMC Studies on Heat Conduction of Gas Confined in a Cuboid Nanopore. Journal of Heat Transfer 139(5): 052405. DOI: 10.1115/1.4035854. 